\title{
TRAFFIC TIME SERIES FORECASTING BY FEEDFORWARD NEURAL NETWORK: A CASE STUDY BASED ON TRAFFIC DATA OF MONROE
}

\author{
M. Raeesi a , M. S. Mesgari a, P. Mahmoudi a \\ ${ }^{a}$ GIS Division and Center of Excellence for Geoinformation Technology. Faculty of Geodesy and Geomatics Engineering, \\ K.N.Toosi University of Technology., Tehran, Iran - moein_3030@yahoo.com, \\ mesgari@kntu.ac.ir, payammahmoudi89@gmail.com
}

KEY WORDS: Traffic, Neural networks, Time series forecasting, Intelligence Transportation System

\begin{abstract}
:
Short time prediction is one of the most important factors in intelligence transportation system (ITS). In this research, the use of feed forward neural network for traffic time-series prediction is presented. In this paper, the traffic in one direction of the road segment is predicted. The input of the neural network is the time delay data exported from the road traffic data of Monroe city. The time delay data is used for training the network. For generating the time delay data, the traffic data related to the first 300 days of 2008 is used. The performance of the feed forward neural network model is validated using the real observation data of the 301 st day.
\end{abstract}

\section{INTRODUCTION}

\subsection{Motivation of the study}

One of the most important activities related to traffic control is the planning for short-term forecasting, an example of which can be the prediction of daily traffic for the next few days. Prediction of traffic can be used to improve the traffic condition and reduces travel time having the available capacity. Prediction system uses the emerging computer, communication and control technologies for managing and monitoring the transportation. Many factors such as weather condition, exhibitions and holidays can affect the quality of the traffic forecasting. One of the prediction methods is the time-series forecasting. In time-series forecasting, the historical data are collected and analyzed to make a model. Then this model is extrapolated for forecasting the future values (Zhang, 2012).

\subsection{Research objectives}

In recent decades, neural networks have been used increasingly for modeling complex phenomena. Neural networks have a high potential to design model and predict the future data compared to traditional time-series models (Balkin and Ord, 2000). This research aims at modeling the time-series forecasting using feed-forward neural network.

\subsection{Overview of the related work}

Many traditional methods are developed for time-series prediction (Ljung and Box, 1978). However, in recent decades, neural networks have often been used for modeling the prediction (Gershenfeld and Weigend, 1994). He and Lapedes (1994) studied the nonlinear time-series by using neural networks. Time-series prediction can be done using both linear and nonlinear methods. Linear method can be implemented and developed easily. Moreover, they are more understandable and interpretable than nonlinear methods. Nevertheless, this methods has limitation: they cannot capture nonlinear relations and their approximation is not as easy as nonlinear ones (Liang, 2005). Medeiros et al. (2001) used neural networks for modeling the linear time-series. de Groot and Würtz (1991) used feed forward neural network for two benchmark nonlinear time-series.Atiya et al. (1999) present a multistep river flow forecasting. Berardi and Zhang (2003) determined the bias and variance issue in time-series forecasting context. Liang (2005) used Bayesian neural networks for time-series analysis. Balkin and Ord (2000) suggested the neural networks for large scale time-series forecasting. Adya and Collopy (1998) reported the application of time-series forecasting by neural networks.

\section{METHODOLOGY}

Describing the methodology of this research consists of two main parts. In the first part, the general theoretical foundation of neural networks is described. In the next part, the usage of neural networks for time series forecasting is described. Figure 1 shows the main steps of this research.

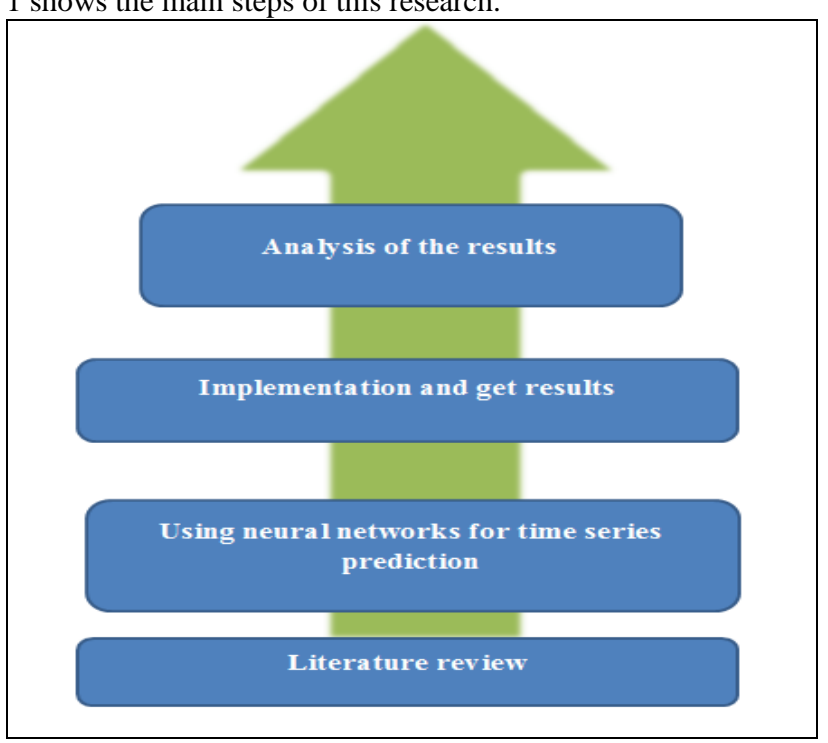

Figure1. Methodology flowchart 


\subsection{Neural network}

Neural networks are computing models to compute the process of information. They are useful for recognizing the pattern or relationship between data (Zhang, 2012). A network is a collection of simple structures which together create a complex system. There are different types of networks, all of which are composed of two components:

1. The set of nodes, each node in the network computing unit which performs processing on the input and output obtained.

2. Links between nodes which define the connections and the transition of the data between nodes. These connections can be unidirectional or bidirectional.

Interaction between nodes through the network connections are presented by a general behavior. Such behavior does not occur alone in any of the network elements. Extensive application of the general behavior on each node makes a network a powerful tool. The neural networks have three segments: input layer, hidden layer and output layer as showed in the following figure.

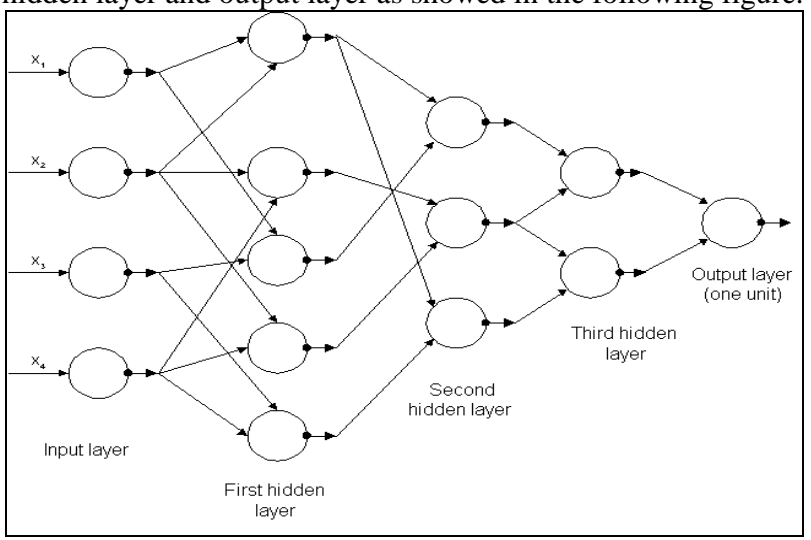

Figure 2. An example of a neural network

Error back propagation algorithm is a method of network training which modifies the network weights. This method is used in the feed forward Neural Networks. Feed forward means that the outputs of the neurons in each layer are transferred to the next layer. To implement this approach, the network weights are randomly selected then based on input and output data, errors are calculated. Weights are updated in the sense that the calculated errors are minimized.

\subsection{Using neural network for time-series forecasting}

For solving different problems, many type of neural network are developed. For time-series forecasting, the feed forward neural network is mostly used. The following figure represents a feed forward neural network. This kind of neural network is composed of several layers called nodes or neurons. In this network model, there is one input layer and one output layer. The input nodes are used for receiving the data of the past. For the time-series prediction, $\left(\mathrm{y}_{\mathrm{t}} \mathrm{y}_{\mathrm{t}-\mathrm{q}} \ldots \mathrm{y}_{\mathrm{t}-\mathrm{p}}\right)$ are used as inputs. The most important part of the network is the hidden layer. Hidden layer is composed of nodes which are connected to both output and input layers. The output nodes are used for prediction of the future value of the time-series. In the feed forward neural network, the information flow is in one directional.

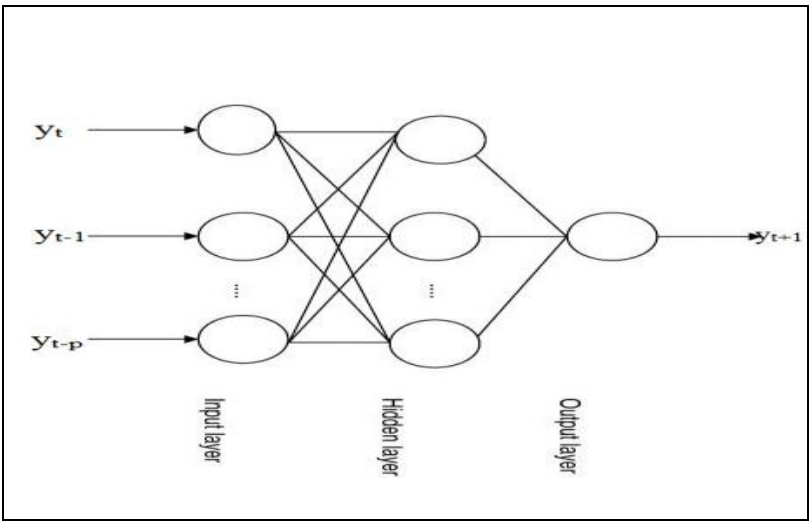

Figure 3. Feed forward neural networks for time series forecasting

This feed forward neural network is functionally equivalent to a nonlinear model, such as the following,

$Y_{t+1}=f\left(y_{t}, y_{t-1}, \cdots y_{t-p}\right)+\varepsilon_{t+1}$

Where $\mathrm{y}_{\mathrm{t}}$ is the observed time series value at time $\mathrm{t}$ and $\varepsilon_{t+1}$ is the error term at time $t+1$. This model shows that the future value of time-series $\left(\mathrm{Y}_{\mathrm{t}+1}\right)$ is a function of the past observations $\left(\mathrm{y}_{\mathrm{t}} \mathrm{y}_{\mathrm{t}-1} \ldots \mathrm{y}_{\mathrm{t}-\mathrm{p}}\right)$ with a random error (Zhang, 2012). This kind of models assumes that there is a relationship between future value and the past observation and neural networks are used for identifying this relationship.

\section{IMPLEMENTATION AND RESULTS}

\subsection{Case study and data set}

The study area is Monroe city in the state of Louisiana. Monroe is the eighth-largest city of the U.S. For recording the traffic of this city, the data of permanent and temporary stations are used. We used the traffic data of 2008. In addition, the data of permanent station with station-ID 430110 is used. This station records the traffic data each hour. Therefore, 8784 data element is recorded. The following figure shows the location and the specification of the station.

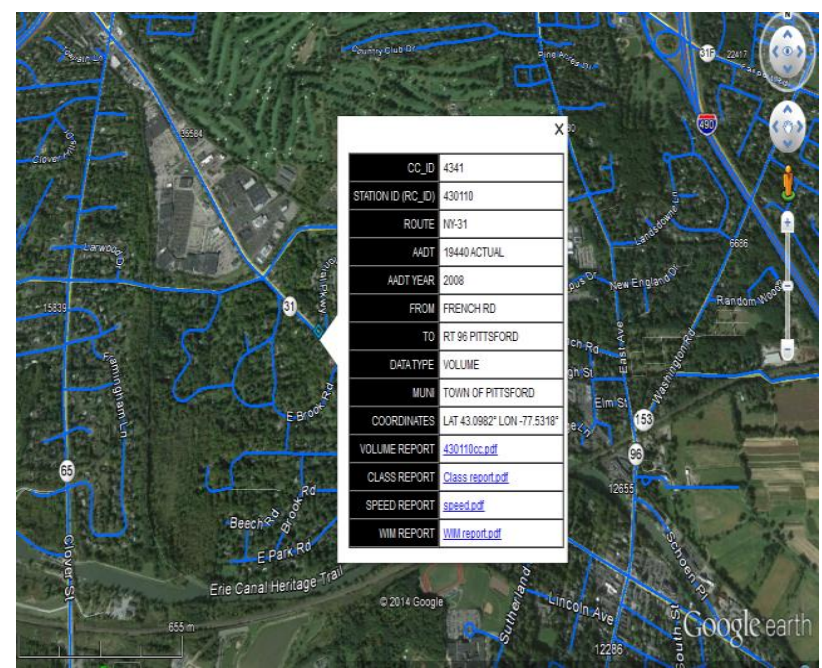

Figure 4. The location and specification of the traffic station

For our research, we used one direction of the road for timeseries forecasting. The format of data is shown in figure 5 . 


\begin{tabular}{|c|r|r|r|r|}
\hline \hline & A & B & C & \multicolumn{1}{c|}{ D } \\
\hline 1 & station number & dierection & traffic & start time \\
\hline 2 & 430110 & 3 & 95 & $1 / 1 / 20081: 00$ \\
\hline 3 & 430110 & 3 & 56 & $1 / 1 / 20082: 00$ \\
\hline 4 & 430110 & 3 & 35 & $1 / 1 / 20083: 00$ \\
\hline 5 & 430110 & 3 & 14 & $1 / 1 / 20084: 00$ \\
\hline 6 & 430110 & 3 & 9 & $1 / 1 / 20085: 00$ \\
\hline 7 & 430110 & 3 & 11 & $1 / 1 / 20086: 00$ \\
\hline 9 & 430110 & 3 & 31 & $1 / 1 / 20087: 00$ \\
\hline 10 & 430110 & 3 & 31 & $1 / 1 / 20088: 00$ \\
\hline 11 & 430110 & 3 & 37 & $1 / 1 / 20089: 00$ \\
\hline 12 & 430110 & 3 & 52 & $1 / 1 / 200810: 00$ \\
\hline 13 & 430110 & 3 & 98 & $1 / 1 / 200811: 00$ \\
\hline 14 & 430110 & 3 & 140 & $1 / 1 / 200812: 00$ \\
\hline 15 & 430110 & 3 & 178 & $1 / 1 / 200813: 00$ \\
\hline 16 & 430110 & 3 & 203 & $1 / 1 / 200814: 00$ \\
\hline 18 & 430110 & 3 & 224 & $1 / 1 / 200815: 00$ \\
\hline 19 & 430110 & 3 & 225 & $1 / 1 / 200816: 00$ \\
\hline 20 & 430110 & 3 & 244 & $1 / 1 / 200817: 00$ \\
\hline 21 & 430110 & 3 & 181 & $1 / 1 / 200818: 00$ \\
\hline 22 & 430110 & 3 & 176 & $1 / 1 / 200819: 00$ \\
\hline 23 & 430110 & 3 & 127 & $1 / 1 / 200820: 00$ \\
\hline & 430110 & 3 & 116 & $1 / 1 / 200821: 00$ \\
\hline
\end{tabular}

Figure 5 .Format of the traffic data

\subsection{Implementation}

Neural network is trained using the data of the first 300 days of the year and the traffic of the 301st day is predicted using this network. We assume that the traffic condition for tomorrow is a function of the traffic of today, yesterday, one week ago, two weeks ago, three weeks ago and four weeks ago at the same time. Therefore, the network has 6 inputs and one output. In this case, the model of equation can be like equation 2 .

$$
Y_{t+1 \text { day }}=f\left(y_{t}, y_{t-1 \text { day }}, y_{t-7 \text { day }}, y_{t-14 d a y}, y_{t-21 \text { day }}, y_{t-28 d a y}\right)+\varepsilon_{t+1}
$$

$\mathrm{y}_{\mathrm{t}}$ is the observed traffic value of today and $\mathrm{y}_{\mathrm{t}-1 \mathrm{day}}$ is traffic data of yesterday and so on and $\mathrm{Y}_{\mathrm{t}+1 \mathrm{lday}}$ is the traffic of tomorrow. With this assumption, inputs and output of the neural network are like figure 6 .

\begin{tabular}{|c|c|c|c|c|c|c|}
\hline$y_{t}$ & $\mathrm{y}_{\mathrm{t}-\mathrm{ld} a \mathrm{y}}$ & $\mathrm{y}_{\mathrm{t}-7 \mathrm{~d} a \mathrm{y}}$ & $y_{t-14 d a y}$ & $y_{t-21 d a y}$ & $\mathrm{y}_{\mathrm{t}-28 \mathrm{day}}$ & $Y_{t+1 \text { day }}$ \\
\hline 37 & 32 & 42 & 38 & 47 & 95 & 42 \\
\hline 15 & 10 & 13 & 10 & 29 & 56 & 18 \\
\hline 6 & 6 & 11 & 11 & 5 & 35 & 5 \\
\hline 6 & 5 & 5 & 4 & 4 & 14 & 6 \\
\hline 5 & 5 & 5 & 6 & 3 & 9 & 5 \\
\hline 7 & 7 & 4 & 7 & 7 & 11 & 4 \\
\hline 27 & 26 & 25 & 24 & 21 & 31 & 30 \\
\hline 111 & 107 & 101 & 96 & 100 & 31 & 112 \\
\hline 251 & 242 & 261 & 255 & 260 & 37 & 231 \\
\hline 248 & 260 & 225 & 248 & 235 & 52 & 260 \\
\hline 220 & 224 & 217 & 205 & 224 & 98 & 212 \\
\hline 251 & 262 & 266 & 258 & 254 & 140 & 238 \\
\hline 328 & 314 & 334 & 281 & 337 & 178 & 269 \\
\hline 375 & 364 & 365 & 367 & 400 & 203 & 342 \\
\hline 369 & 358 & 377 & 345 & 376 & 224 & 337 \\
\hline 399 & 373 & 366 & 352 & 411 & 225 & 368 \\
\hline 376 & 392 & 418 & 408 & 415 & 244 & 357 \\
\hline 415 & 406 & 409 & 415 & 473 & 181 & 403 \\
\hline 430 & 425 & 440 & 434 & 439 & 176 & 410 \\
\hline 347 & 343 & 343 & 363 & 342 & 127 & 321 \\
\hline
\end{tabular}

Figure 6 .input and output data of neural network

In this research, $80 \%$ of the data is used for training of network and $20 \%$ of the data is used for testing the network. The hidden layer of the neural network has three layers. There are 10 neurons in layer one, 15 neurons in layer two, and one neuron in the last layer. Hybrid tangent sigmoid is used for transfer function. Figure 7 shows the architecture of the neural network.

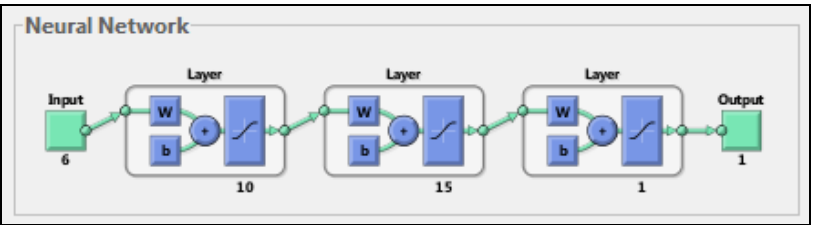

Figure7. Architecture of the neural network

\subsection{Result}

The mean square error of the training data is 0.0229 . Figure 8 shows the procedure of convergence of mean square error of training data.

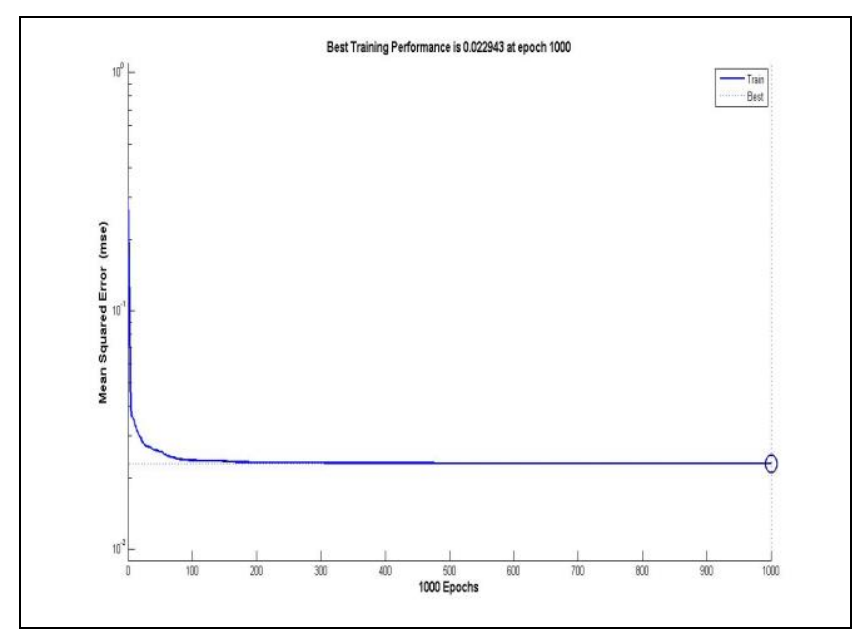

Figure 8. Procedure of convergence

Comparison between the real values of the traffic and the results obtained from the network are presented in Figure 9. The red cycles are the training data and the blue squares are the output training data of the neural network.

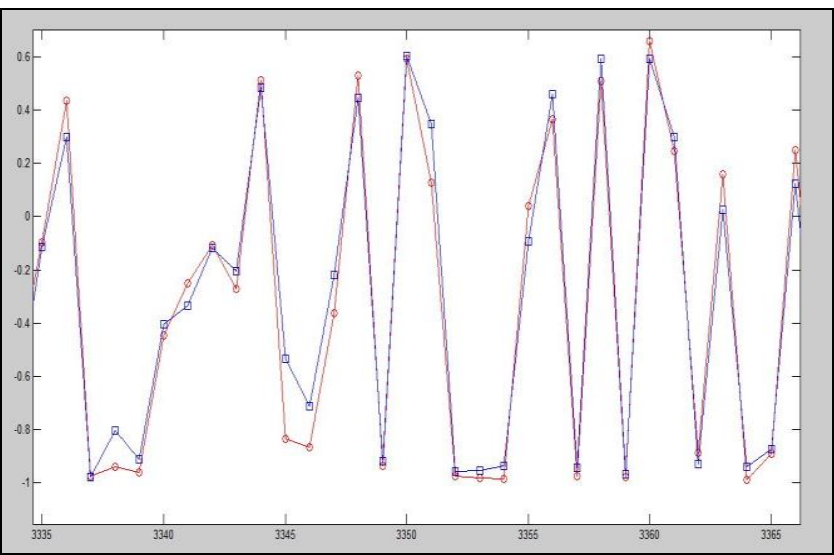

Figure 9. Results of the neural network for the training data

The mean square error for the test data which is not used for constructing the network is 0.0345 . The results for the test data is presented in Figure 10. In this figure, the red cycles are the test data and the blue squares are the output test data of the neural network. 


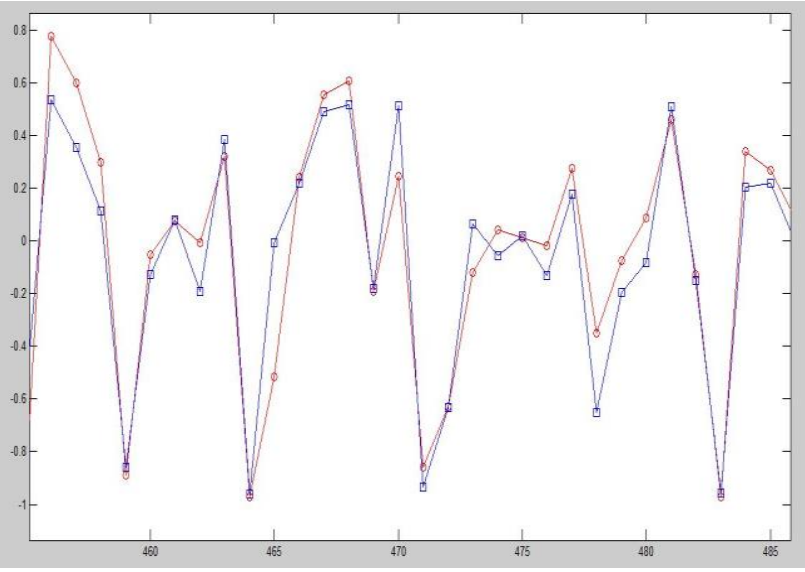

Figure 10. Results of the neural network for the test data

If the output of the network and the corresponding observed are closed, the network training is acceptable. In other words, if we have a plot with training data on $\mathrm{X}$ axis and output of neural network for training data on $\mathrm{Y}$ axis, the results are near to diagram $\mathrm{y}=\mathrm{x}$, as shown in figure 11 . This is true for test data as well, as shown in figure 12 .

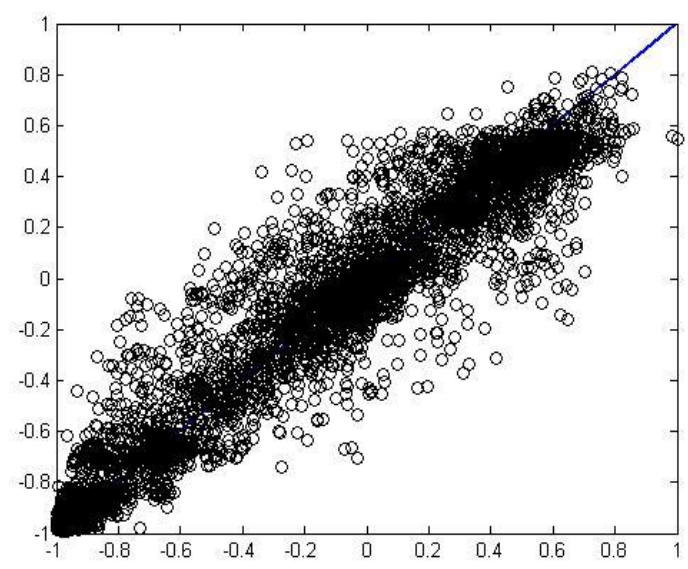

Figure11. Results of training and neural network for training data

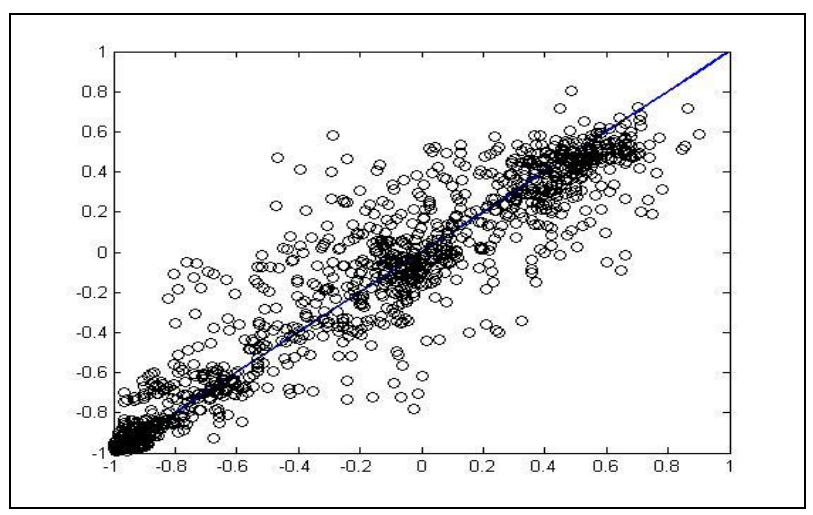

Figure12. Results of test and neural network for test data

We use this neural network for prediction of the 301st day and compare it with the real number of traffic data as shown in table 1.

\begin{tabular}{|c|c|c|}
\hline $\begin{array}{l}\text { Time step } \\
\text { prediction }\end{array}$ & prediction & actual \\
\hline 24 & 26 & 34 \\
\hline 23 & 74 & 71 \\
\hline 22 & 187 & 150 \\
\hline 21 & 91 & 110 \\
\hline 20 & 291 & 259 \\
\hline 19 & 309 & 340 \\
\hline 18 & 281 & 300 \\
\hline 17 & 356 & 400 \\
\hline 16 & 362 & 405 \\
\hline 15 & 373 & 373 \\
\hline 14 & 358 & 380 \\
\hline 13 & 338 & 353 \\
\hline 12 & 377 & 356 \\
\hline 11 & 313 & 270 \\
\hline 10 & 177 & 180 \\
\hline 9 & 72 & 100 \\
\hline 8 & 178 & 190 \\
\hline 7 & 43 & 103 \\
\hline 6 & 11 & 29 \\
\hline 5 & 5 & 9 \\
\hline 4 & 5 & 6 \\
\hline 3 & 23 & 25 \\
\hline 2 & 31 & 11 \\
\hline 1 & 75 & 50 \\
\hline
\end{tabular}

Table 1. Prediction for 24 hours of the 361 st day

Finally, the root square error between prediction values and actual values is 26.4889 which is acceptable for such a prediction.

\section{CONCLUSION AND DISCUSSION}

In this study, feed forward neural network is used for timeseries forecasting of the traffic related to the 301st day of 2008. We design this neural network for predicting one direction of a road segment. The proposed neural network used the traffic data of today, previous day, one week ago, two weeks ago, three weeks ago and four weeks ago at the same time for predicting tomorrow traffic volume at that time. Some large error can be resulted in the cases of car accidents, bad weather etc.

We used above parameters for inputs of neural network. However, to improve the prediction, auto correlation of the data can be derived by some methods like Cochrane-Orcutt. For future work, the traffic of all segments can be predicted.

\section{REFERENCES}

Adya, M., Collopy, F., 1998. How effective are neural networks at forecasting and prediction? A review and evaluation. $J$. Forecasting 17, 481-495.

Atiya, A.F., El-Shoura, S.M., Shaheen, S.I., El-Sherif, M.S., 1999. A comparison between neural-network forecasting techniques-case study: river flow forecasting. Neural Networks, IEEE Transactions on 10, 402-409.

Balkin, S.D., Ord, J.K., 2000. Automatic neural network modeling for univariate time series. International Journal of Forecasting 16, 509-515. 
Berardi, V.L., Zhang, G.P., 2003. An empirical investigation of bias and variance in time series forecasting: modeling considerations and error evaluation. Neural Networks, IEEE Transactions on 14, 668-679.

de Groot, C., Würtz, D., 1991. Analysis of univariate time series with connectionist nets: A case study of two classical examples. Neurocomputing 3, 177-192.

Gershenfeld, N., Weigend, A., 1994. The Future of Time Series: Learning and Understanding. Time Series Prediction: Forecasting the Future and Understanding the Past. Addison Wesley, New York.

He, X., Lapedes, A., 1994. Nonlinear modeling and prediction by successive approximation using radial basis functions. Physica D: Nonlinear Phenomena 70, 289-301.

Liang, F., 2005. Bayesian neural networks for nonlinear time series forecasting. Statistics and Computing 15, 13-29.

Ljung, G.M., Box, G.E., 1978. On a measure of lack of fit in time series models. Biometrika 65, 297-303.

Medeiros, M.C., Veiga, A., Pedreira, C.E., 2001. Modeling exchange rates: smooth transitions, neural networks, and linear models. Neural Networks, IEEE Transactions on 12, 755-764.

Zhang, G.P., 2012. Neural Networks for Time-Series Forecasting, in: Rozenberg, G., Bäck, T., Kok, J. (Eds.), Handbook of Natural Computing. Springer Berlin Heidelberg, pp. 461-477. 